\title{
Spin-selective transport through helical molecular systems
}

\author{
R. Gutierrez, ${ }^{1}$ E. Díaz, ${ }^{1,2}$ R. Naaman, ${ }^{3}$ and G. Cuniberti ${ }^{1,4}$ \\ ${ }^{1}$ Institute for Materials Science, Dresden University of Technology, D-01062 Dresden, Germany \\ ${ }^{2}$ Grupo Interdisciplinar de Sistemas Complejos (GISC), Departamento de Física de Materiales, Universidad Complutense, \\ E-28040 Madrid, Spain \\ ${ }^{3}$ Department of Chemical Physics, Weizmann Institute, 76100 Rehovot, Israel \\ ${ }^{4}$ Division of IT Convergence Engineering National Center for Nanomaterials Technology, Pohang University of Science and Technology \\ (POSTECH), Pohang 790-784, Republic of Korea
}

(Received 1 February 2012; published 16 February 2012; corrected 8 May 2012)

\begin{abstract}
Highly spin-selective transport of electrons through a helically shaped electrostatic potential is demonstrated in the frame of a minimal model approach. The effect is significant even for weak spin-orbit coupling. Two main factors determine the selectivity: an unconventional Rashba-like spin-orbit interaction, reflecting the helical symmetry of the system, and a weakly dispersive electronic band of the helical system. The weak electronic coupling, associated with the small dispersion, leads to a low mobility of the charges in the system and allows even weak spin-orbit interactions to be effective. The results are expected to be generic for chiral molecular systems displaying low spin-orbit coupling and low conductivity.
\end{abstract}

DOI: 10.1103/PhysRevB.85.081404

PACS number(s): 72.25.-b, 73.22.-f, 73.63.-b, 87.85.J-

Introduction. The concept of spintronic devices operating without a magnetic field has been proposed some time ago for solid state devices in which the spin-orbit coupling (SOC) is large. ${ }^{1,2}$ In recent years, a different type of magnet-less spinselective transmission effect has been reported. ${ }^{3-7}$ It was found that electron transmission through self-assembled monolayers (SAMs) of chiral molecules is highly spin selective at room temperature. These findings are so far surprising as organic molecules typically have a small SOC that cannot support significant splitting between the spin states. Although it has been suggested both by theory ${ }^{8,9}$ and experiments ${ }^{10}$ that there is a cooperative contribution to the value of the SOC, making this quantity larger in molecules or nanotubes than in a single carbon atom, the values calculated or experimentally found are still relatively small, ${ }^{8-11}$ e.g., a few meV for nanotubes. ${ }^{10}$ Hence, even including this cooperative contribution, the spin polarization (SP) in electron transmission through SAMs of chiral molecules ${ }^{6,7}$ seems to be too high to be rationalized by such SOC values.

Recently, a theoretical model based on scattering theory has been proposed for explaining the spin selectivity of chiral molecules. ${ }^{12}$ Although the results are in qualitative agreement with the experimental observations, they could not explain them by using reasonable SOC values.

In what follows, a minimal model is presented, describing electron transmission through a helical potential—see Fig. 1. The main goal of the model is to highlight the role of some crucial parameters, which will lead to a high SP while still keeping a moderate SOC strength. Although the recent transport experiments on DNA $\mathrm{SAMs}^{7}$ are our main motivation, the model is generic enough to encompass other molecular systems with chiral symmetry.

In short, there are two main key factors in the model allowing for a high SP: (i) Lack of inversion symmetry due to the chiral symmetry of the scattering potential, and (ii) narrow electronic bandwidths in the helical system, i.e., the coupling between the units composing the helical structure is relatively weak. A physically meaningful estimation of the
SOC is further obtained by taking into account that first, in the present study, the electric field acting on the electron needs to include the effective influence of all the electrons belonging to a molecular unit, ${ }^{7,13}$ and second, due to proximity effects, the Coulomb interaction between the transmitted electron and those in the molecular unit scales as $1 / R$ for short distances $R$.

Model and methodology. We consider the Schrödinger equation for a particle moving in a helical electrostatic field. Analytical results for such fields have been derived in Ref. 14. For the sake of simplicity, approximate expressions valid near the $z$ axis will be used (only $x, y$ components will be considered; the $z$ component only contributes when considering the full three-dimensional problem): $\mathbf{E}_{\text {helix }}=$ $-E_{0} \sum_{l, j} g_{l, j}(z)[\cos (Q j \Delta z), \sin (Q j \Delta z)]$. Here, $g_{l, j}(z)=$ $\left\{1+[(z-l b-j \Delta z) / a]^{2}\right\}^{-3 / 2}$, and $Q=2 \pi / b$, with $b$ being the helix pitch and $a$ the helix radius-see Fig. 1 . The index $j=0, \ldots, M_{0}-1$ runs along one helical turn and labels the $z$ coordinate of the $M_{0}$ molecular units placed along one turn of the helix. The index $l=-L_{0} / 2, \ldots, L_{0} / 2\left(L_{0}\right.$ being the number of helical turns) connects sites which differ in their $z$ coordinate by $b .{ }^{15}$ We note that the considered helical potential is assumed to be related to the charge distribution along the stack of molecular units building the helical structure; hence the factor $E_{0}$ is proportional to the local charge density.

For a charge moving with momentum $\mathbf{p}$ through the helix, the field $\mathbf{E}_{\text {helix }}$ induces a magnetic field in the charge's rest frame, leading to a SOC: $H_{\text {SO }}=\lambda \sigma\left(\mathbf{p} \times \mathbf{E}_{\text {helix }}\right)$. The SOC strength is $\lambda=e \hbar /(2 m c)^{2}$ and $\sigma$ is a vector whose components are the Pauli matrices $\sigma_{x}, \sigma_{y}$, and $\sigma_{z}$. The general problem is three dimensional (3D); however, in order to get insights into the behavior of the SP, we will assume $p_{x}=p_{y}=0, p_{z} \neq 0$, so that the Schrödinger equation takes the form ${ }^{16}$

$$
\begin{array}{r}
{\left[-\frac{\hbar^{2}}{2 m} \partial_{z}^{2}+U(z)+\alpha\left(\begin{array}{cc}
0 & \Psi(z) \\
-\Psi^{*}(z) & 0
\end{array}\right) \partial_{z}\right.} \\
\left.+\alpha\left(\begin{array}{cc}
0 & f(z) \\
-f^{*}(z) & 0
\end{array}\right)\right] \chi(z)=E \chi(z) .
\end{array}
$$




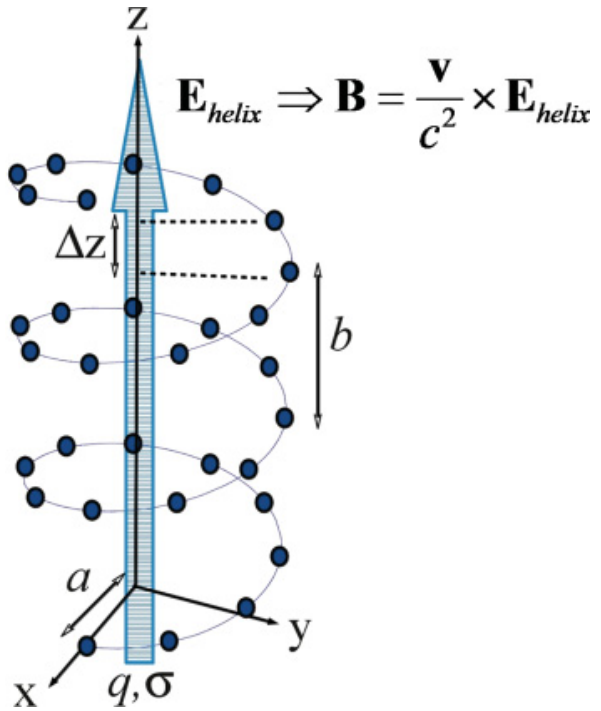

FIG. 1. (Color online) A charge $q$ in spin state $\sigma$ is moving along through a helical electric field. The parameters $a, b$, and $\Delta z$ are the radius and the pitch of the helix and the spacing of the $z$ component of the position vector of the charges distributed along it, respectively. The helical field $\mathbf{E}_{\text {helix }}$ induces a magnetic field $\mathbf{B}$ in the rest frame of the charge and hence influences its spin state.

Here, $\chi(z)=\left[\chi^{\uparrow}(z), \chi^{\downarrow}(z)\right]^{T}$ is a spinor, $\Psi(z)=-\left[E_{x}-\right.$ $\left.i E_{y}\right] / E_{0}=\sum_{l, j} e^{-i Q j \Delta z} g_{l, j}(z), f(z)=\partial_{z} \Psi(z)$, and $U(z)$ the helical electrostatic potential. ${ }^{16}$ The terms $\sim f(z), f^{*}(z)$ are introduced to make the Hamiltonian hermitian in the continuum representation. The SOC parameter $\alpha=\hbar \lambda E_{0}$ (with dimensions of energy $\times$ length) depends on the effective charge density through $E_{0}$. The problem posed by Eq. (1) can be written as an effective two-channel tight-binding model:

$$
\begin{aligned}
H= & \sum_{\sigma=\uparrow, \downarrow} \sum_{n=1}^{N} U_{n} c_{n, \sigma}^{\dagger} c_{n, \sigma}+V \sum_{\sigma=\uparrow, \downarrow} \sum_{n=1}^{N-1}\left(c_{n, \sigma}^{\dagger} c_{n+1, \sigma}+\text { H.c. }\right) \\
& +\sum_{n, m=1}^{N}\left(c_{n, \uparrow}^{\dagger} W_{n, m} c_{m, \downarrow}+c_{m, \downarrow}^{\dagger} W_{m, n}^{\times} c_{n, \uparrow}\right)+H_{\text {leads. }}
\end{aligned}
$$

The operators $\left\{c_{n, \sigma}, c_{n, \sigma}^{\dagger}\right\}_{n=1, \ldots, N, \sigma=\uparrow, \downarrow}$ create or destroy, respectively, an excitation at the tight-binding site $n$ with spin index $\sigma$. The only nonzero elements of the interchannel coupling matrix $\mathbf{W}$ are given by $W_{n, n}=-\alpha f(n \Delta z), W_{n, n+1}=$ $\alpha \Psi(n \Delta z) / 2 \Delta z$, and $W_{n+1, n}=-\alpha \Psi((n+1) \Delta z) / 2 \Delta z .{ }^{16}$ Further, the matrix $W_{n, m}^{\times}$satisfies $W_{n, m}^{\times}=-\left(W_{m, n}\right)^{*}$ for $n \neq m$, and $W_{n, n}^{\times}=\left(W_{n, n}\right)^{*}$, which reflects time inversion symmetry in the system. The hopping $V$ can in general be estimated via a first-principles calculation of the electronic structure for a given system. Here, it will be considered as a free parameter, whose order of magnitude for helical organic systems is expected to lie in the range of a few tens of meV [e.g., for DNA, $V \sim 20-40 \mathrm{meV}$ (Refs. 17 and 18)]. Finally, the operator $H_{\text {leads }}$ includes the semi-infinite chains to the left and right of the SO active region. ${ }^{16} \mathrm{~A}$ schematic representation of the model is shown on the top panel of Fig. 2.

Transport properties. We focus on the spin-dependent transmission probability $T(E)$ of the model given by Eq. (2), as a function of the electron's injection energy $E$. The
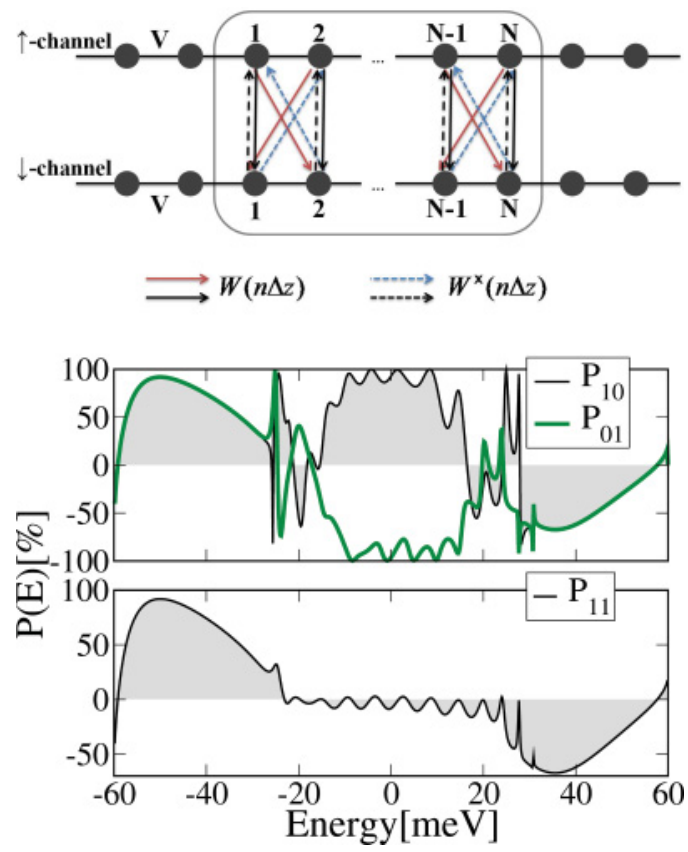

FIG. 2. (Color online) Top: Schematic representation of the tightbinding model-see Eq. (2). The two channels interact via the SOC (framed region). To the left and right of the spin scattering region, both channels are independent and are modeled by semi-infinite chains. Bottom: Energy dependence of the SP $P(E)$ for $L_{0}=3$ helical turns, and for injected electrons polarized with their spin pointing up $\left(P_{10}\right)$, down $\left(P_{01}\right)$, or unpolarized $\left(P_{11}\right)$. A spin-filter effect takes place only for energies near the band edges, where all SPs have the same sign. Notice also that near the band edges the SP has opposite signs for electrons $(E<0)$ and holes $(E>0)$, though $P(E)$ is not exactly antisymmetric. Parameters: $\alpha=5 \mathrm{meV} \mathrm{nm}, V=30 \mathrm{meV}$, $U_{0}=3 \mathrm{meV}$.

problem can be considered as a scattering problem where a finite-size region (with nonvanishing SOC) is coupled to two independent $L$ (left) and two independent $R$ (right) electrodes, each electrode standing for a spin channel and being represented by a semi-infinite chain-see Fig. 2. $T(E)$ encodes the influence of multiple scattering events in the SOC region. We assume a coherent transport regime and use Landauer's theory ${ }^{19}$ to obtain ${ }^{16}$

$$
\begin{aligned}
T(E)= & \Gamma_{\uparrow}^{R}\left(\Gamma_{\uparrow}^{L}\left|G_{1 \uparrow, N \uparrow}\right|^{2}+\Gamma_{\downarrow}^{L}\left|G_{1 \downarrow, N \uparrow}\right|^{2}\right) \\
& +\Gamma_{\downarrow}^{R}\left(\Gamma_{\uparrow}^{L}\left|G_{1 \uparrow, N \downarrow}\right|^{2}+\Gamma_{\downarrow}^{L}\left|G_{1 \downarrow, N \downarrow}\right|^{2}\right) \\
= & t_{\text {up }}(E)+t_{\text {down }}(E) .
\end{aligned}
$$

In Eq. (3), $G_{n \sigma, m v}(E)$ are matrix elements of the retarded Green's function of the SOC region including the influence of the $L$ and $R$ electrodes. Each contribution in Eq. (3) can be related to a different transport process without (e.g., $\Gamma_{\uparrow}^{L} \Gamma_{\uparrow}^{R}\left|G_{1 \uparrow, N \uparrow}\right|^{2}$ ) or with (e.g., $\Gamma_{\uparrow}^{L} \Gamma_{\downarrow}^{R}\left|G_{1 \uparrow, N \downarrow}\right|^{2}$ ) spin-flip scattering. Notice that $t_{\mathrm{up}}(E)$ and $t_{\text {down }}(E)$ - the transmissions for the up and down channels, respectively-contain contributions arising both from direct transmission without spin flip as well as spin flip. An energy-resolved SP for different initial spinor states can be defined as $P(E)=$ $\left[t_{\text {up }}(E)-t_{\text {down }}(E)\right] / T(E)$. The energy-average $\mathrm{SP}\langle P(E)\rangle_{E}=$ $P\left(\left\langle t_{\text {up }}(E)\right\rangle,\left\langle t_{\text {down }}(E)\right\rangle,\langle T(E)\rangle\right)$ will also be used. We focus 
only on electronlike contributions $(E<0)$ and on energies $|E| \geqslant k_{B} T \approx 23 \mathrm{meV}$, so that $\langle\cdots\rangle_{E}=\int_{-2 V}^{-k_{B} T} d E(\cdots)$.

Results. A crucial parameter in the model is the SOC coupling $\alpha$. Realistic values are obviously very difficult to obtain, ${ }^{20,21}$ since $\alpha$ is not simply the atomic SOC, but contains the influence of the charge distribution in the system via the field factor $E_{0}$. For the sake of reference, a rough value of $E_{0}$ for DNA may be estimated along the following lines. A single DNA base is considered as composed of discrete pointlike charge centers $A$ representing the atoms. We associate with each center $A$ at position $R_{A}$ a Gaussian-shaped charge distribution of width $w \sim 0.3-0.4 \mathrm{~nm}$ and with strength given by an estimated atomic charge density $\rho_{0}$ for $\mathrm{C}, \mathrm{N}$, and $\mathrm{O}$ atoms (considered as spheres with a radius of the order of the corresponding covalent radius). The local field of this charge distribution, $E_{0}=-\left(1 / 4 \pi \epsilon_{0}\right)(\partial / \partial r) \int d^{3} r^{\prime} \rho\left(r^{\prime}-\right.$ $\left.R_{A}\right)\left|r-r^{\prime}\right|^{-1}$, can be computed analytically ${ }^{16}$ and it scales for $R=\left|r-R_{A}\right| \ll w$ as $E_{0} \approx\left(N_{0} \rho_{0} / 4 \pi \epsilon_{0}\right)(w / 2 \sqrt{\pi})^{2} R^{-1}$ ( $E_{0}$ has been multiplied by a factor $N_{0} \sim 10$, the number of atoms in a base, to approximately account for other charge centers). For $R / w \sim 0.3-0.4$, values of $\alpha=\hbar \lambda E_{0} \approx$ 1.87-2.35 $\mathrm{meV} \mathrm{nm}$ are obtained. In the calculations, $\alpha \sim$ 2-6 meV $\mathrm{nm}$ is used. Though this analysis only provides a very rough estimate, it highlights the need for considering the influence of many charges through $\rho_{0}$ and $N_{0}$ as well as proximity effects (short-distance scaling of $E_{0}$ ) in the estimation of $\alpha$.

Figure 2 presents the energy-dependent SP for $\alpha=$ $5 \mathrm{meV} \mathrm{nm}$, and for different incoming spin states: spin up (10), spin down (01), or unpolarized electrons (11). In the case of (10) and (01) states, the interesting energy windows are those where both SPs have the same sign, indicating that the outgoing state will always have the same SP independently of the initial condition. This behavior occurs mainly for energies near the band edges. A similar situation is found for the (11) state-see Fig. 2. Near the band center, $P_{10}(E)$ and $P_{01}(E)$ have opposite signs and hence the SP depends on the incoming spin state. The average SP, as defined above, amounts to approximately $\left\langle P_{10}\right\rangle_{E}=\left\langle P_{01}\right\rangle_{E}=\left\langle P_{11}\right\rangle_{E} \approx 62 \%$.

Figure 3 shows the corresponding spin-resolved transmissions. In the top panel of Fig. 3, for (10) and (01), we overall find some degree of spin-dependent backscattering, which is reflected in different total transmissions $T_{10}(E) \neq T_{01}(E)$. In what follows, for the sake of reference, only the behavior in the energy window $\left[-2 V,-k_{B} T\right], k_{B} T \sim 23 \mathrm{meV}$ is discussed. For the (10) state, transmission without spin flip is dominant in this energy region, and this leads to the positive SP. On the contrary, for (01) spin-flip processes become dominant in the same energy region, and hence the outgoing up channel acquires a larger weight. As a result, the SP for (01) is also positive. This behavior is closely related to the chiral symmetry, which basically manifests in the special structure of the $W, W^{\times}$ matrices. For the (11) state (the bottom panel of Fig. 3), the outgoing up channel clearly dominates the transmission in the considered energy window, thus indicating that for unpolarized electrons backscattering and spin-flip of the down component will also lead to a positive SP. In general, SP may occur either by spin flip (with no net change of total transmission) or by spin-selective backscattering: The previous discussion suggests that both processes play a role here.

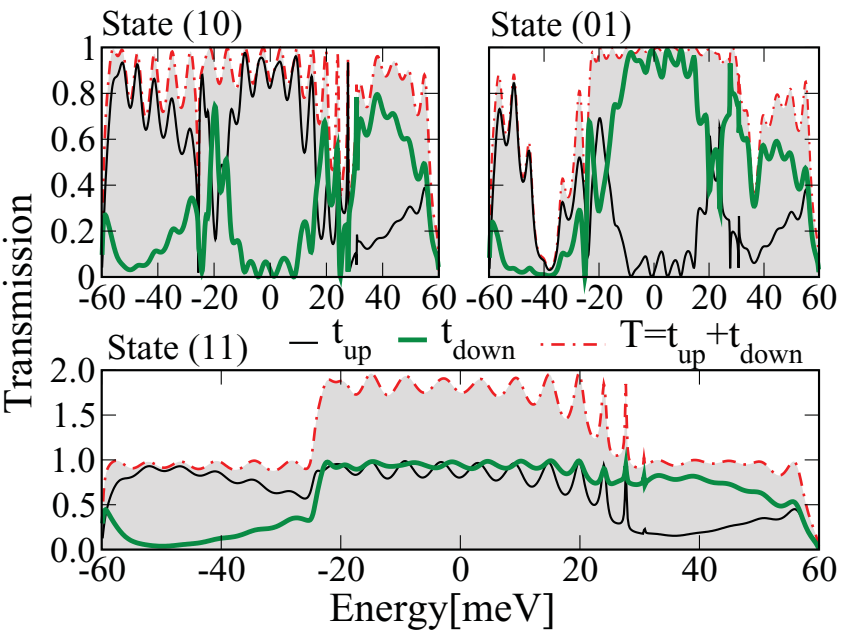

FIG. 3. (Color online) Different components of the transmission $t_{\text {up }}(E), t_{\text {down }}(E)$, and $T(E)$ as defined in Eq. (3), and for the same parameters of Fig. 2. Focusing on electronlike contributions, it is only near the lower band edge $(E \leqslant-22 \mathrm{meV})$ where a positive SP for all incoming states (10), (01), and (11) is obtained-see also Fig. 2.

The found selectivity relates to two special features of the chiral system: (i) the helical field symmetry which translates into an unconventional SOC, and (ii) the weak electronic coupling $V$. As shown in Fig. 4, the size of the hopping parameter strongly affects the energy average SP, ultimately leading to $\langle P(E)\rangle_{E} \rightarrow 0$ for large $V$. For small hopping, however, the SP can achieve very large values by only a moderate increase of the $\operatorname{SOC} \alpha$. The interplay between $\alpha$ and $V$ seems related to the relatively long time $\tau \sim \hbar V^{-1}$ the electron will spend in the conducting channel in a real system, allowing a weak SOC to become more effective.

Conclusions. The present study, which is based on a minimal model, sheds light on a strong chirality-induced spin-selectivity (CISS) effect. It suggests that CISS may be a generic phenomenon, existing in chiral systems having low SOC and low conductivity, and hence may play a role in spin

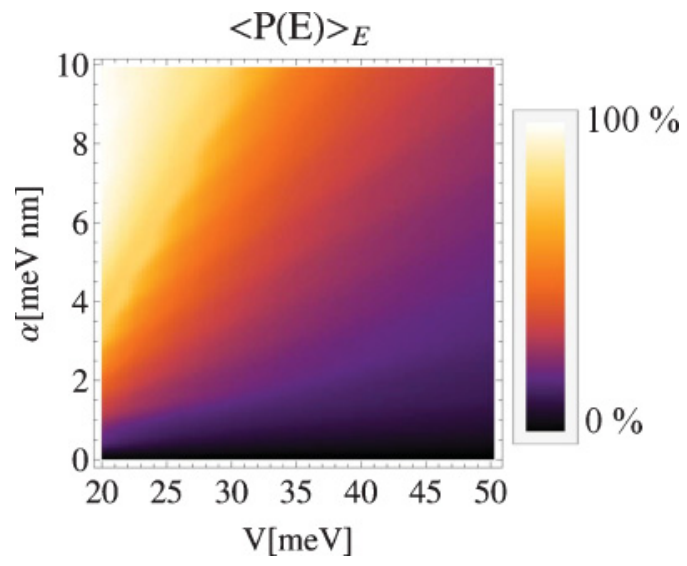

FIG. 4. (Color online) Two-dimensional (2D) plot of the energy average SP $\langle P(E)\rangle_{E}$ as a function of both the hopping parameter $V$ and the SOC $\alpha$. Only for small $V$ a relative large SP is found. With increasing electronic coupling, larger SOC strengths are required to get a sizable SP. 
transport through biosystems. Weak electronic coupling along the helical structure is expected to lead to low mobility of the electrons and to allow enough time for the SOC, although being weak, to influence spin transport. The effect depends on the electron momentum and once the electrons have kinetic energy above $k_{B} T$, the SP increases and becomes weakly energy dependent. We remark that the inclusion of static on-site disorder weakens, but does not suppress, the effect, as long as the disorder strength remains smaller than $V .^{16}$ We may find this situation realized in the studied SAMs of chiral molecules, ${ }^{6,7}$ where geometric constraints (steric hindrance, coupling to the substrate) can be expected to largely quench conformational disorder. One open issue for further inquiry is the influence of the electrode-molecule interface. If the electrodes are magnetic, spin-dependent tunnel barriers may influence the SP. The found CISS effect could also be of great interest to control the spin injection efficiency in the context of semiconductor-based spintronics by interfacing chiral molecules with semiconductor materials.

Acknowledgements. R.G. and E.D. thank H. Pastawski, R. Bustos-Marun, T. Brumme, and S. Avdoshenko for fruitful discussions. This work was partially funded by the DFG under CU 44/20-1, MAT2010-17180, and by the South Korea Ministry of Education, Science, and Technology Program "World Class University" (No. R31-2008-000-10100-0). Computational resources were provided by the $\mathrm{ZIH}$ at TU-Dresden. E.D. thanks Ministerio de Educación y Ciencia (MEC) and R.N. thanks the German-Israel Science Foundation and the Israel Science Foundation for financial support. Travel between Dresden and Madrid was supported by the Ministerio de Economia y Comptetitividad (project PRI-AIBDE-20110927) and by the German Academic Exchange Service (project 54367888) within the joint program Acciones Integradas.
${ }^{1}$ B. Datta and S. Das, Appl. Phys. Lett. 56, 665 (1990).

${ }^{2}$ J. P. Lu, J. B. Yau, S. P. Shukla, M. Shayegan, L. Wissinger, U. Rössler, and R. Winkler, Phys. Rev. Lett. 81, 1282 (1998).

${ }^{3}$ K. Ray, S. P. Ananthavel, D. H. Waldeck, and R. Naaman, Science 283, 814 (1999).

${ }^{4}$ R. Naaman and Z. Vager, MRS Bull. 35, 429 (2010).

${ }^{5}$ S. G. Ray, S. S. Daube, G. Leitus, Z. Vager, and R. Naaman, Phys. Rev. Lett. 96, 036101 (2006).

${ }^{6}$ B. Goehler, V. Hamelbeck, T. Z. Markus, M. Kettner, G. F. Hanne, Z. Vager, R. Naaman, and H. Zacharias, Science 331, 894 (2011).

${ }^{7}$ Z. Xie, T. Z. Markus, S. R. Cohen, Z. Vager, R. Gutierrez, and R. Naaman, Nano Lett. 11, 4652 (2011).

${ }^{8}$ D. Huertas-Hernando, F. Guinea, and A. Brataas, Phys. Rev. B 74, 155426 (2006).

${ }^{9}$ A. De Martino, R. Egger, K. Hallberg, and C. A. Balseiro, Phys. Rev. Lett. 88, 206402 (2002).

${ }^{10}$ F. Kuemmeth, S. Ilani, D. C. Ralph, and P. L. McEuen, Nature (London) 452, 448 (2008).
${ }^{11}$ E. I. Rashba, Sov. Phys. Solid State 2, 1109 (1960) [Fiz. Tverd. Tela (Leningrad) 2, 1224 (1960)].

${ }^{12}$ S. Yeganeh, M. A. Ratner, E. Medina, and V. Mujica, J. Chem. Phys. 131, 014707 (2009).

${ }^{13}$ G. Bihlmayer, S. Blügel, and E. V. Chulkov, Phys. Rev. B $\mathbf{7 5}$, 195414 (2007).

${ }^{14}$ D. Hochberg, G. Edwards, and Th. W. Kephart, Phys. Rev. E 55, 3765 (1997).

${ }^{15}$ We chose for the sake of reference $b \sim 3.2 \mathrm{~nm}, a \sim 0.7 \mathrm{~nm}$, and $\Delta z=0.32 \mathrm{~nm}$, values which are close to those of B-DNA.

${ }^{16}$ See Supplemental Material at http://link.aps.org/supplemental/ 10.1103/PhysRevB.85.081404 for further details.

${ }^{17}$ P. B. Woiczikowski, T. Kubar, R. Gutierrez, G. Cuniberti, and M. Elstner, J. Chem. Phys. 130, 215104 (2009).

${ }^{18}$ R. Gutierrez, R. A. Caetano, B. P. Woiczikowski, T. Kubar, M. Elstner, and G. Cuniberti, Phys. Rev. Lett. 102, 208102 (2009).

${ }^{19}$ A. Nitzan, Annu. Rev. Phys. Chem. 52, 681 (2001).

${ }^{20}$ C. L. Kane and E. J. Mele, Phys. Rev. Lett. 95, 226801 (2005).

${ }^{21}$ Z. G. Yu, Phys. Rev. Lett. 106, 106602 (2011). 